\title{
Constructing a Model for Creating Movie Trailers Increase Customers’ Desire
}

\author{
Kazuka Yanagisawa, ${ }^{1, *}$, Takayuki Iida ${ }^{1} \&$ Kakuro Amasaka ${ }^{1}$ \\ ${ }^{1}$ Aoyama Gakuin University, Sagamihara-shi, Japan \\ *Corresponding author: Aoyama Gakuin University, 5-10-1, Fuchinobe, Chuo-ku, \\ Sagamihara-shi, Kanagawa-ken, 252-5258, Japan. Tel: 81-42-759-6423. E-mail: \\ c5612133@aoyama.jp
}

Received: November 17, 2013 Accepted: January 8, 2014 Published: January 20, 2014 doi:10.5296/rae.v6i1.4945 URL: http://dx.doi.org/10.5296/rae.v6i1.4945

\begin{abstract}
Nowadays, movie box-office revenue and movie theater visitor numbers are relatively stagnant. A particular problem is that there are fewer movie goers among young people in their early twenties to early thirties, who tend to spend more. In order to solve this problem, it is important to use advertising to increase customers' desire to watch movies, and movie trailers in particular play a significant role. Currently, the production of movie trailers is very subjective and relies on experience and intuition. It is a matter of concern that the relative quality of movie trailers is inconsistent and, furthermore, qualitative evaluations are ambiguous (implicit). Thus, this research aims to create movie trailers that will increase customers' desire to watch movies by using statistical science and biometrics. Specifically, this involves (1) investigating the compositional elements of movie trailers, (2) conducting tests using electrodermal responses, (3) analysing lines of sight during viewing of movie trailers, (4) analysing the scenes composing the trailer using pair comparison, and (5) measuring brain activity during viewing of movie trailers. Based on the findings, the authors constructed a "movie trailer scene composition model" by quantitatively clarifying factors such as when and what kind of scenes should be incorporated into movie trailers. This model has been used to create movie trailers that will increase customers' desire to watch movies, and the required results have been attained.
\end{abstract}

Keywords: compositional elements model of movie trailers; electro dermal responses; measuring brain activity 


\section{Introduction}

Moviemaking has become a major industry in Japan. Due to factors such as the emergence of multi-screen cinema complexes, the number of movie theater screens and the number of movies shown have both been increasing in recent years. By contrast, growth in box office revenue and movie theater visitor numbers has slowed and leveled off since 2001, making it necessary for today's industry to improve the quality of its advertising to increase the customers' desire to watch movies.

Currently, movie trailers are created by movie trailer producers based on the concept of the film. However, the creative process is a subjective one that depends on the producer's intuition and technique, and the quality of the resulting trailer varies significantly with the producer who created it. This study aims to propose a movie trailer scene compositional model by quantitatively assessing the characteristics of movie trailers that increase the viewers' desire to watch movies and to facilitate the creation of trailers that increase that desire based on that model.

\section{State of the Movie Industry in Japan}

Growth in box office revenue and movie theater visitor numbers has been flat in recent years (Japanese Movie Industry Statistics, 2012). Advertising that increases the customers' desire to watch movies will be important in resolving this issue, and movie trailers have a major role to play. Since production of movie trailers is a subjective process characterized by significant variations in quality, the ambiguity of assessments of their quality remains an outstanding problem. Compared to box office revenue of $¥ 170.862$ billion and theater attendance of 1,353.9 million in 2000, revenue and attendance have shown growth, reaching highs of $¥ 220.737$ billion and 1,743.58 million in 2010. Yet figures for 2011 show a decrease in both metrics, with revenue falling to $¥ 181.197$ billion and attendance to $1,447.26$ million. By contrast, the number of films showing exceeded 800 in 2006 and, despite a subsequent slight decline, was still 799 in 2011, 100 films more than around 2003. The number of screens, which was about 2,500 in 2000, exceeded 3,000 in 2006 and reached about 3,400 in 2011, indicating an increasing trend in recent years. Simply increasing the number of films and screens is not sufficient to boost box office revenue and movie theater visitor numbers.

Examining the movie participation rate by age, the authors find that it is higher for younger consumers and that it decreases with age. However, looking at how that rate has changed over time, the authors find that while the participation rate for teenagers and customers in their twenties has fallen over the last 10 years, the rate has increased for customers aged 40 and higher. Nonetheless, the participation rate for young consumers is higher, and that group comprises a major segment of the movie market.

Taking all of this together, theaters have put in place an environment in recent years that allows customers to watch more movies. However, movie theater visitor numbers are hovering around 1.6 billion, and the authors can infer that simply increasing the number of films and screens is not sufficient in order to encourage customers to watch more movies. In 
the current state of affairs, there will be fewer and fewer opportunities to have customers watch movies if their interest is not awakened, and simply producing more movies will not accomplish this objective.

Compounding this challenge is a trend among young consumers, who constitute a major segment of the movie market, to seek entertainment elsewhere than from movies. Consequently, the contemporary movie industry finds itself faced with the need to increase the quality of its advertising to increase the customers' desire to watch movies.

\section{Previous Research}

The field of film is starting to attract scientific attention, as evidenced by research by Takahashi et al. on movie titles (2000) and by Tanimura on causes of movie-related behavior (2004). This research concluded that AIDA theory, a behavior model, applies to the process extending from recognition of a movie to the behavior of going to a theater, even though movies are an intangible asset. Imamura demonstrated that it is possible to assess the emotional state of customers watching movies in a quantitative manner by means of the SAM method (2007). Shinosaki and Shiizuka conducted a sensibility-based evaluation of movie posters (2007), and Uchida, Ohara and Yamada developed an approach for increasing customers' desire to watch movies using the same media (2009), demonstrating the effectiveness of applying statistical science and line-of-sight analysis to the creation of movie posters. Koyama focused on the responsibilities of movie producers, who play an important role in the production of movies, and offered them creative support in order to improve the quality of movies by focusing on movie planning (2010).

Based on this information, the authors focused on movie trailers based on our belief that they, out of all the movie industry's advertising media, are best suited to attract the attention of the general public and communicate information about movies due to their use of video content.

As an example of previous research that has targeted movie trailers, Shu-Gao Ma and Wei-Qiang Wang (2011) and Iida, Goto, and Fukuchi cited the effectiveness of using GSR (Galvanic Skin Response) biometrics in the creation of movie trailers (2011). This study focuses on video as a characteristic of movie trailers and, in an effort to build on this research, the authors have sought to clarify, quantify, and quantitatively assess the relationship between time and scenes by using the same statistical science techniques employed by Amasaka et al. as well as brain activity measurement techniques utilized by Kono and Muranaka (2010) in order to develop a creative support approach model for movie trailer producers.

\section{Creating Movie Trailers that Increase the Desire to Watch Movies}

\subsection{Interviews of Workers at a Movie Trailer Production Company}

To investigate the movie trailer production process, the authors interviewed workers at Gal Enterprise Inc. They found that implicit in the work is an approach to trailer composition that 
relies on the underlying movie's concept and that trailer prototypes are ty'pically created and revised numerous times during the production process. Since creators rely on their own intuition, techniques, and experience to do their work in a subjective manner, differences among creators lead to differences in the quality of the trailers.

In order to achieve the objective of the movie trailer that is to increase customers' desire to watch the movie, it becomes necessary to make a quantitative assessment of what types of trailer will successfully increase customers' interest in watching movies. Since these interviews led us to an understanding that customer preferences and required elements differ with the movie genre and customer age group, for this study the authors opted to focus on action movies and on young consumers, the largest market for movies and yet one that is moving away from films.

\subsection{Types of Composition in Movie Trailers}

To clarify the nature of the compositional elements in movie trailers that increase the customers' desire to watch movies, the authors investigated the importance of a variety of variables such as the presence of stylistic rhythm and introduction of the cast (Figure 1.).

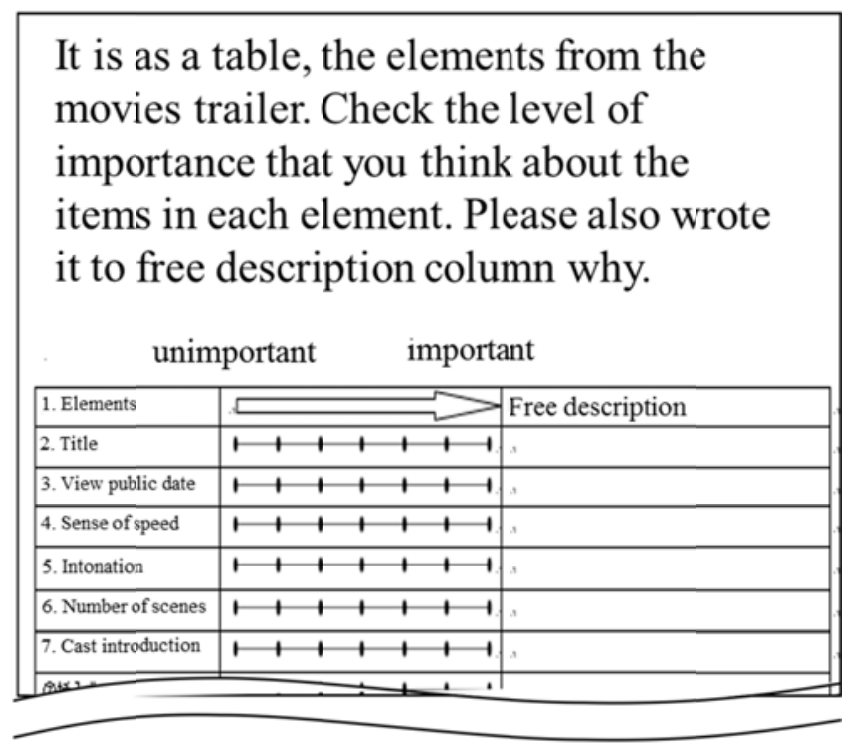

Figure 1. An Example of a Questionnaire

\subsection{Analysis of Compositional Elements in Movie Trailers}

The authors conducted a factor analysis to assess the relationships between movie trailer compositional elements in a more specific manner. We were able to do so in this analysis by grouping the 17 trailer compositional elements according to common factors and clarifying the common factors operating among the elements.

They named the No. 1 factor "Evaluation”, which was characterized by high factor loadings in the form of movie festival prizes, industry rankings, and customers' abillity to imagine their feelings after watching the movie. Similarly, they named the No. 2 factor "Story" based on plot outline, accessibility, and climactic scene. They named the No. 3 factor "Video" based on stylistic rhythm, pace, and the number of scenes. The No. 4 factor was named "Creators" 


\section{MInstitute Macrothink $_{\text {Int }}$}

based on the distributor, director, and cast. The No. 5 factor was named "Role" based on indication of the opening date, indication of the title, and message. The No. 6 factor was named "Narration" and the No. 7 factor was named "Soundtrack" (Figure 2.).

The authors made a numerical assessment of the relationships among movie trailer compositional elements by conducting a covariance structure analysis to weight the elements (Figure 3.). In this way, it was found that the elements of particular importance were "Soundtrack," indication of title and opening date in "Role of the Trailer," accessibility and plot outline in "Movie Story," and stylistic rhythm in "Trailer Video."

\begin{tabular}{|c|c|c|c|c|c|c|c|}
\hline & \multicolumn{7}{|c|}{ 因子 } \\
\hline & 1 & 2 & 3 & 4 & 5 & 6 & 7 \\
\hline Film festival prize & 777 & -.101 & 017 & .051 & .169 & .013 & .015 \\
\hline Box office & .709 & -.002 & .240 & .193 & -.120 & .173 & .213 \\
\hline Feeling after the movie & 518 & 337 & .078 & .157 & -.094 & .183 & .106 \\
\hline Outline & .029 & .750 & -0.055 & -.126 & .119 & .148 & .170 \\
\hline Plaimness & .054 & 633 & .090 & -.121 & -.180 & -.005 & .033 \\
\hline Climax scene & -.089 & 452 & .026 & .127 & -.124 & -.099 & -.200 \\
\hline Modulation & .134 & -.104 & 931 & -011 & -.021 & .135 & .040 \\
\hline Speed & .072 & 087 & 379 & -.235 & -.084 & -.071 & -.001 \\
\hline Scene number & .188 & 200 & 334 & .085 & .171 & -.237 & .224 \\
\hline Company name & .227 & -.110 & -.186 & 814 & .086 & .039 & -.167 \\
\hline Director name & .264 & -.063 & -.102 & 661 & .061 & -.031 & .216 \\
\hline Cast introduction & -.061 & 052 & .223 & 285 & .049 & -.010 & .024 \\
\hline Public date & .025 & -.108 & 234 & .124 & 904 & -.029 & -.067 \\
\hline Title & .023 & -020 & -090 & .045 & .405 & .046 & -044 \\
\hline Message & .068 & .233 & .261 & .028 & -376 & .288 & -.069 \\
\hline Narration & .231 & .039 & -.004 & .009 & .058 & 895 & .198 \\
\hline Вем & .172 & .025 & .064 & .051 & -.143 & .157 & 827 \\
\hline
\end{tabular}

Figure 2. Factor Matrix after Rotation

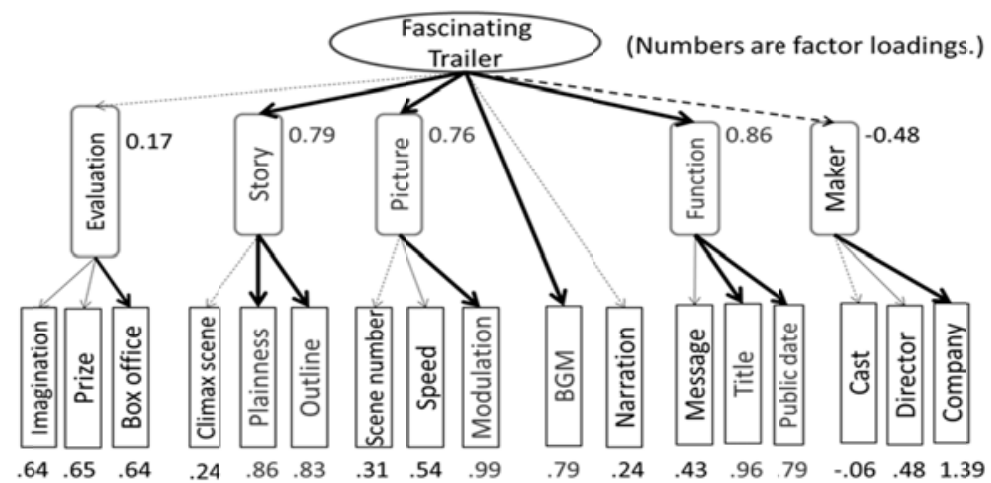

Figure 3. Model Diagram of the Movie Trailer Important Element

It was identified characteristics of each trailer compositional element based on free-response answers concerning the elements' importance (Figure 4.). Using text mining, it was able to articulate the specific roles of the elements. 


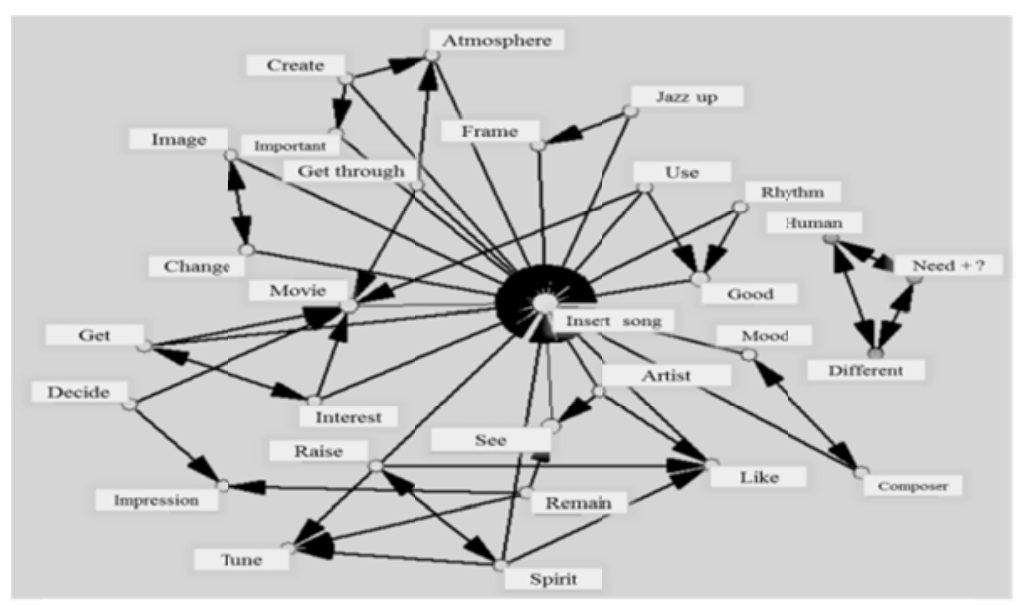

Figure 4. Result of Text Mining

\subsection{Visualization of Emotion with GSR (Galvanic Skin Response) Biometrics}

By using GSR, it is possible to assess changes in the viewers' mental state, such as those that occur when they are excited or thrilled, by measuring changes in the electrical resistance of the skin caused by emotional sweating. Emotional sweating is most likely to be expressed at the fingertips, and when watching a movie trailer, a mental sweating reaction appears at the fingertips as viewers gain visual and auditory information and stimulation. In this study, the authors measured the reactions of 13 subjects as they watched movie trailers for three films: The Expendables, The Insitemill, and Eat Pray Love (Figure 5.). It was measured the difference in GSR before and after the subjects watched the trailers and calculated the mean and standard deviation for these values. For the purposes of our experiment, we then defined values in excess of the reaction boundary value of $\{$ Reaction boundary value $=($ Mean +2$) \times$ standard deviation $\}$. In this way, the authors assessed the reactions of subject No. 1 through No. 13 (11 of whom provided valid data) as they watched the three movie trailers as a time sequence (Figure 6.).

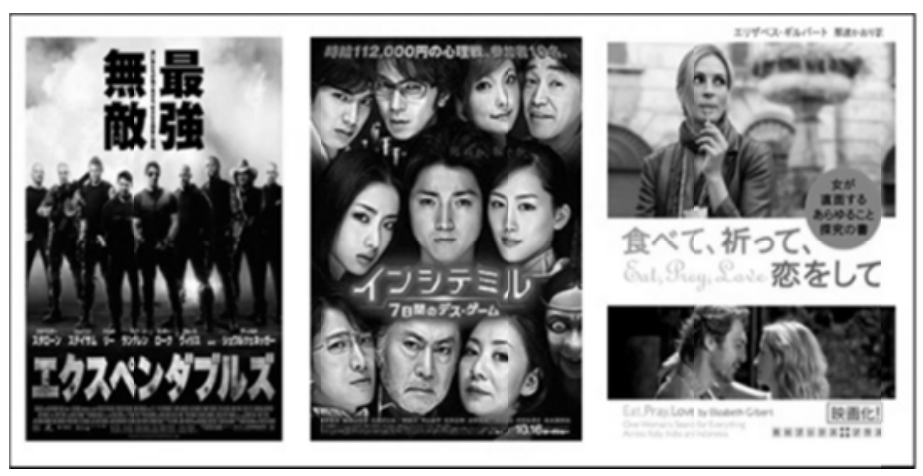

Figure 5. 3 Movies Selected as Experimental 


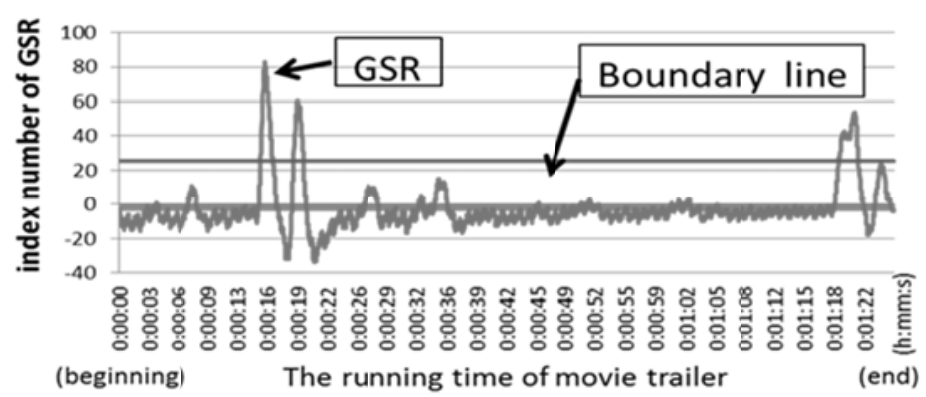

Figure 6. GSR Result (Expendables)

Figure 7. below lists the number of subjects exhibiting reactions in line with time sequences for each of the movie trailers. Only times at which three or more subjects exhibited a reaction are included, and times at which four or more subjects exhibited a reaction have been highlighted.

The Expendables, an action movie, prompted the most reactions. It was also found that overall subjects had the most reactions at the beginning and end of each trailer. It was considered that this fact is linked to the primacy effect and the familiarity effect that is the tendency of the first and last memories, respectively, to remain vivid. Since our aim was to assess which parts of movie trailers elicit viewer excitement using GSR, it was elected to focus on The Expendables, which prompted the most reactions, by conducting a more detailed analysis.

With the goal of clarifying what kind of scenes prompted these reactions, it was posited changes in the brightness of the video image, changes in the soundtrack, and sound effects as the principal causes that influenced viewers' emotional state after conducting a second-by-second comparison of the number of viewers exhibiting a reaction with scene details.

\begin{tabular}{|c|c|c|c|c|c|}
\hline \multicolumn{2}{|c|}{ "Expendables" } & \multicolumn{2}{|c|}{ "Insitemiru" } & \multicolumn{2}{|c|}{ "Eat Pray Love" } \\
\hline Time & Number & Time & Number & Time & Number \\
\hline $0: 01$ & 3 & $0: 05$ & 3 & $0: 01$ & 3 \\
\hline $0: 02$ & 6 & $0: 24$ & 3 & $0: 02$ & 3 \\
\hline $0: 03$ & 6 & $0: 25$ & 3 & $0: 03$ & 3 \\
\hline $0: 04$ & 3 & $0: 26$ & 3 & $0: 47$ & 3 \\
\hline $0: 09$ & 3 & $0: 27$ & 3 & $1: 44$ & 4 \\
\hline $0: 16$ & 4 & $0: 45$ & 5 & $1: 55$ & 4 \\
\hline $0: 19$ & 3 & $0: 46$ & 3 & $2: 08$ & 3 \\
\hline $0: 28$ & 3 & $1: 02$ & 4 & $2: 11$ & 3 \\
\hline $0: 34$ & 3 & $1: 12$ & 4 & & \\
\hline $0: 40$ & 3 & & & \multirow{5}{*}{\multicolumn{2}{|c|}{ Number $>3$}} \\
\hline $0: 58$ & 3 & & & & \\
\hline $1: 07$ & 3 & & & & \\
\hline $1: 20$ & 5 & & & & \\
\hline $1: 21$ & 3 & & & & \\
\hline
\end{tabular}

Figure 7. Reaction Number of People along the Time Series of Each Movie Trailer 


\section{Ml Macrothink}

The authors then developed a time-sequence model for movie trailers that increase the viewers' desire to watch movies based on these experimental results using electrodermal responses (Figure 8.). It was found that with this time-sequence model, which assumes a trailer length of about two minutes, it were able to leave an impression of viewers while increasing their desire to watch the movie in question by moving scenes with a high level of impact to the beginning and the end of the trailer (to take advantage of the primacy and familiarity effects) and placing scenes that introduce the development of the movie's storyline, which were identified as important in our investigation into movie trailer compositional elements, in between.

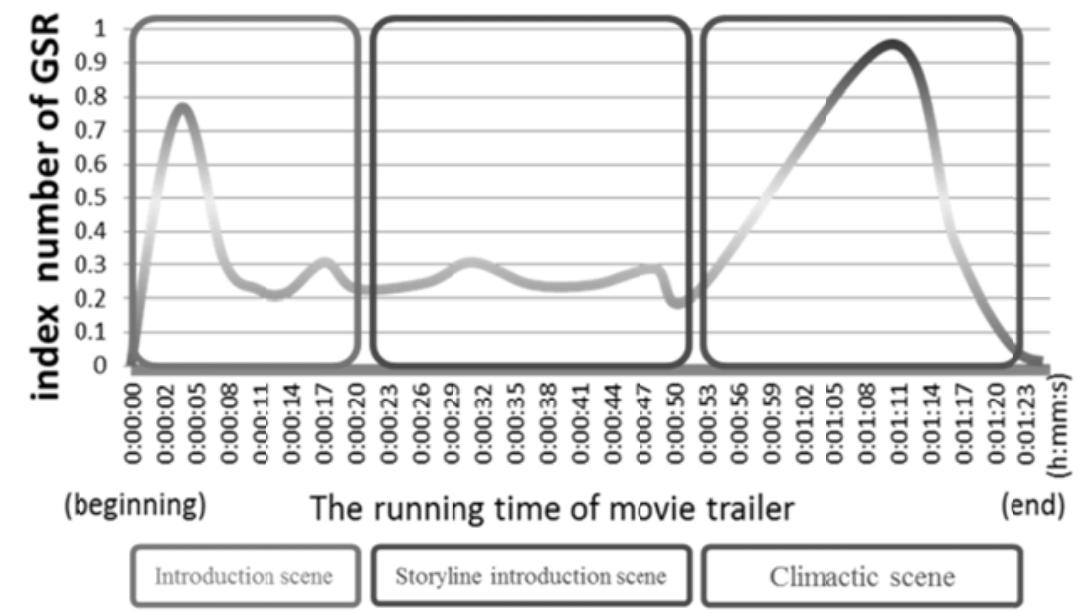

Figure 8. Movie Trailer Time Series Model

\subsection{Analysis of Movie Trailer Scene Composition}

\subsubsection{Analysis of Viewers' Lines of Sight while Watching Movie Trailers}

Using an eye mark recorder, which makes it possible to visualize and measure where viewers are looking, it was identified the elements on which viewers focused their attention while watching the trailer. The authors conducted an AOI analysis to quantify line-of-sight loitering times in order to define a list of the viewers' regions of interest by using heat maps and gaze plots. Heat maps indicate where the viewers' lines of sight are stationary indicating concentration, and gaze plots order the locations at which the subjects' lines of sight loitered. After investigating character and item elements, text and subtitle elements, and background/other, it was found that subjects paid particular attention to character and item elements (Figure 9.). 


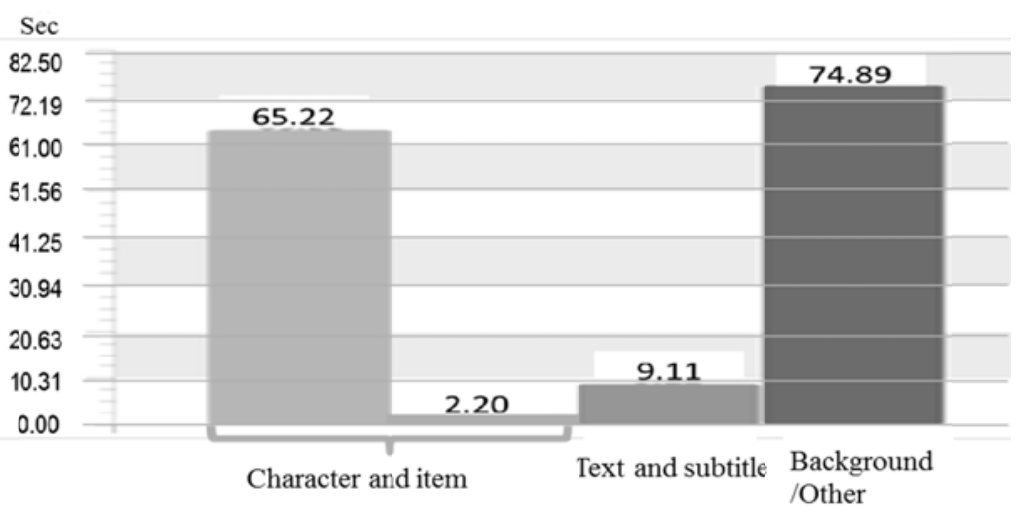

Figure 9. Gaze Dwell Time of AOI

Based on this fact, the authors defined scenes in which character and item elements are active as "Action Scenes," scenes involving dialog instead of character and item action as "Quiet Scenes,” and scenes in which characters and items are absent as "Background."

\subsubsection{Analysis of Trailer Scene Composition Using the Pair Comparison Method}

The authors investigated the optimal proportion of action scenes, quiet scenes, and background in the introduction, storyline introduction, and climactic scene of the trailer and summarized the results in the form of an evaluation using pair comparison (Figure 10.).

\begin{tabular}{|c|c|c|c|c|c|c|c|c|}
\hline \multirow{2}{*}{\multicolumn{2}{|c|}{ Important }} & & \multicolumn{6}{|c|}{$\stackrel{\text { Important }}{\longrightarrow}$} \\
\hline & & \multicolumn{7}{|c|}{ Introduction scene } \\
\hline Action scenes & & & & 8 & 6 & 4 & 1 & Quiet scenes \\
\hline Action scenes & & 1 & 1 & 5 & 6 & 5 & 1 & Background \\
\hline Quiet scenes & & 1 & 2 & & 6 & 3 & & Background \\
\hline & & \multicolumn{7}{|c|}{ Storyline introduction scene } \\
\hline Action scenes & & 1 & 2 & 3 & 5 & 7 & 1 & Quiet scenes \\
\hline Action scenes & & \begin{tabular}{l|l|}
3 & 6 \\
\end{tabular} & 3 & 5 & 2 & & & Background \\
\hline Quiet scenes & & \begin{tabular}{l|l|l}
4 & 7 \\
\end{tabular} & 5 & 2 & & 1 & & Background \\
\hline \multicolumn{9}{|c|}{ Climactic scene } \\
\hline Action scenes & 1 & \begin{tabular}{l|l|}
7 & 7 \\
\end{tabular} & 3 & 1 & & & & Quiet scenes \\
\hline Action scenes & 9 & \begin{tabular}{|l|l|}
6 & 3 \\
\end{tabular} & 1 & & & & & Background \\
\hline Quiet scenes & 1 & \begin{tabular}{|l|l|}
7 & 3 \\
\end{tabular} & 6 & 2 & & & & Background \\
\hline
\end{tabular}

Figure 10. Pairwise Comparison Survey Results

They determined the ratio (standard value) of movie trailer scene composition by assigning a score to each response based on these results. They then divided the difference between the response with the largest sum and that with the smallest sum by the number of responses and calculated the weight of each compositional scene in the introduction, storyline introduction, and climactic scene

\subsubsection{Experiment to Measure Brain Activity while Watching Movie Trailers}

To determine how scenes should be combined, the authors measured brain activity in six subjects as they watched the four target movie trailers. Because our study had identified the skillful implementation of a stylistic rhythm in the video--that is, alternating periods of excitement and relaxation--as important in the creation of a successful movie trailer, they targeted mid-alpha brain waves, which are associated with the subject's being in a relaxed 
state of concentration. Since mid-alpha brain waves can be observed at four frequencies ranging from 9 to $12 \mathrm{~Hz}$, they used the total value of observed voltages from 9 to $12 \mathrm{~Hz}$ in our analysis (Figure 11.). Since values that diverge greatly from the average may be erroneous, they used large reaction boundary values in our effort to assess which types of scene composition allowed viewers to relax and concentrate. It was then developed a number of hypotheses:

- Use of numerous dark scenes is not conducive to a high level of mid-alpha brain activity.

- Sound has a pronounced effect on mid-alpha activity (use of too many loud sounds is not conducive to a high level of mid-alpha activity).

- Use of a series of scenes with intense action or movement is not conducive to a high level of mid-alpha activity.

- Mixing a variety of scenes together is conducive to a high level of mid-alpha activity.

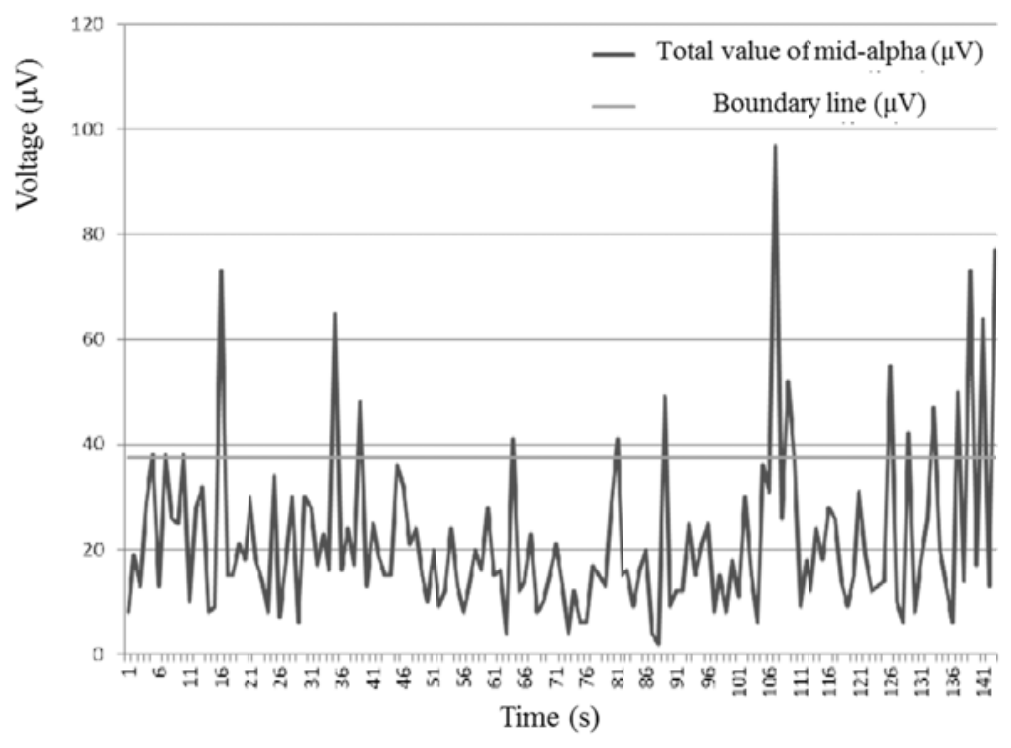

Figure 11. Boundary Line and Total Value of Mid-alpha

\subsubsection{Constructing a Model for Creating Movies Trailers}

It was developed a movie trailer scene composition model based on the information gained from these analyses (Figure 12.). It is not critical to exactly observe the proportion of each scene relative to the whole as obtained from the pair comparison. The authors believe, however, that it is important to come within a margin of $5 \%$ to $10 \%$ of those values. Based on the results of our analysis of movie trailer compositional elements and measurement of the subjects' brain activity as they watched movie trailers, they believe that viewers can be given a strong and favorable impression of the movie by mixing together a number of scenes where possible instead of relying on a monotonous scene composition. This is because giving the trailer stylistic rhythm is particularly important. they believe this can be achieved by placing the introduction of the producer, which isn't particularly important, after the point at which viewers are first excited in the introduction, by placing character and cast introductions at the 
beginning of the storyline introduction, and by placing the title and opening date introductions, which are important elements, immediately after the climactic scene. Based on our electrodermal experiments, careful investigation of the flow of description in movie trailers, and our analysis of the viewer's brain activity, it was set targets of about four seconds for the title introduction, about two seconds for the opening date introduction, and about one second for the production introduction. The cast and character introductions may be lengthened or shortened to reflect the relative importance of those elements in the movie in question. The authors believe that by creating trailers that reflect this movie trailer scene composition model, it will be possible to increase the viewers' desire to watch movies.

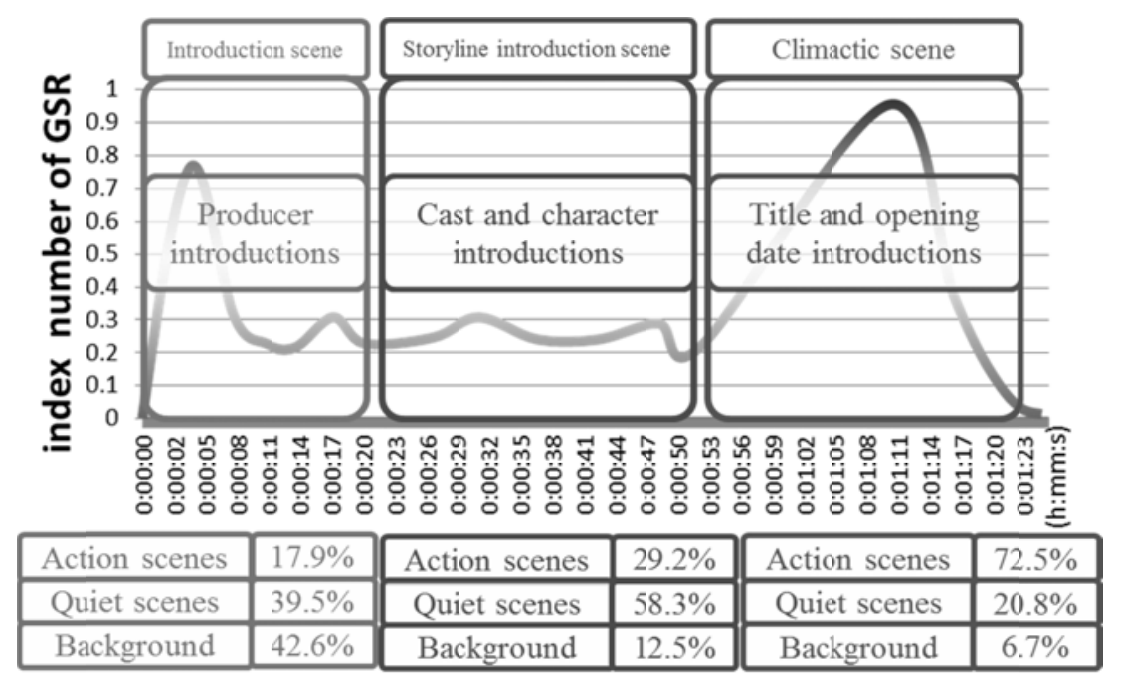

Figure 12. The Movie Trailer Scene Composition Model

\section{Movie Trailer Production and Benefits}

The authors created a new trailer based on the information gained from the movie trailer scene composition model. Based on the facts that it is easiest to obtain reactions for action movies when performing GSR experiments and that our subjects had not seen it, they chose HOT FUZZ - as the target movie.

They conducted a verification using GSR to compare the trailer produced using this model with the existing trailer and gauge whether the viewers' desire to watch the movie had increased and whether the GSR graph had identified as ideal had been achieved. They asked the subjects to watch both the existing trailer and the new trailer, without revealing to them which was which.

The GSR reaction characteristics closely resemble the reaction characteristics we derived as described in Section 5 and could be described as an ideal graph (Figure 13.). 


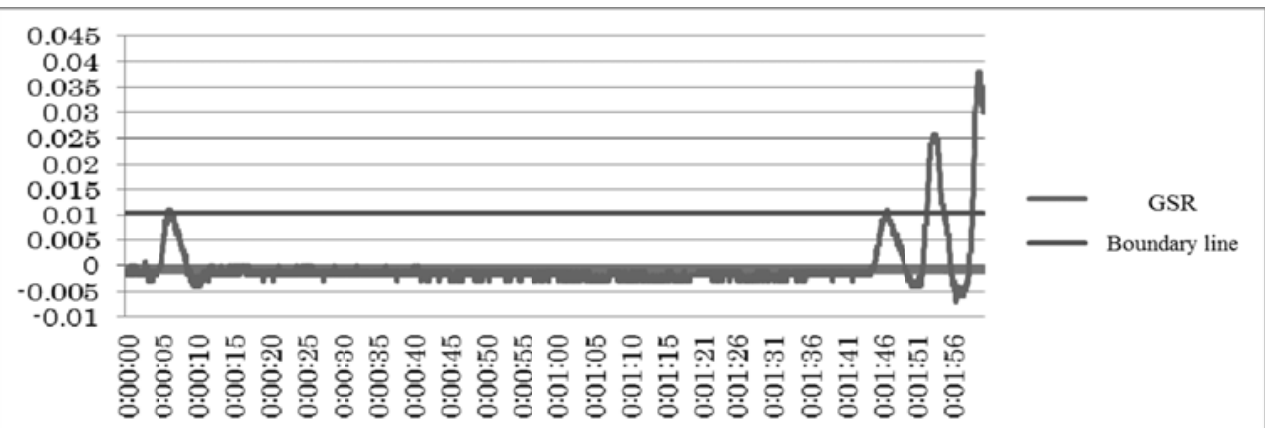

Figure 13. Result of GSR in New Movie Trailer that was Create by the Authors

Below, It was had summarized the reactions of 10 subjects, noting only those locations where at least 3 of the 10 subjects exhibited a reaction and highlighting in red those locations where at least 4 subjects exhibited a reaction. The data reveals that the new trailer prompted more reactions than the existing trailer and that the new trailer triggered a particularly large number of reactions at the beginning and end, confirming the validity of the model.

\section{Conclusion}

In this study, which utilized statistical science and GSR, the authors were able to identify character and item elements using line-of-sight analysis, to develop a movie trailer scene composition model, and to create a movie trailer that increased the viewers' desire to watch the target movie. we conducted a verification using GSR to compare the trailer produced using this model with the existing trailer and gauge whether the viewers' desire to watch the movie had increased and whether the GSR graph had identified as ideal had been achieved. Watching both the existing trailer and the new trailer, without revealing to them which was which, the GSR reaction characteristics closely resemble the reaction characteristics we derived. It means our model has worth when movie trailer creator makes the work, thereby achieving the expected results. In the future, we are going to conduct this model to Japan film industry and want to contribute to attract customers.

\section{References}

Amasaka, K. (2004). Customer science: studying customer values. Japan Journal of Behavior Metrics Society, 32(1), 196-199.

Amasaka, K. (2005). Constructing a Customer Science Application System CS-CIANS Development of a global strategic vehicle Lexus utilizing New JIT -. WSEAS Transactions on Business and Economics, 3(2), 135-142.

Amasaka, K. (2007). The validity of TDS-DTM: A strategic methodology of merchandise development of New JIT -key to the excellence design LEXUS. International Business \& Economics Research Journal, 6(11), 105-116. 
Iida, T., Goto, A., Fukuchi, S., \& Amasaka, K. (2011). A study on an effectiveness of movie trailers boosting customer's appreciation desire: Using statistics and GSR. Aoyama Gakuin Universtiy's graduation thesis. (Unpublished)

Imamura, M. (2007). A study to capture the hearts of customers in watching movies :The approach model proposed by the SAM method. Aoyama Gakuin Universtiy's graduation thesis. (unpublished)

Kono, Y., \& Muranaka, N. (2008). Estimation of brain activity area on sensitivity evaluation by mesuring EEG. Technical Committee on Nurocomputing (NC) by IEICE , 110, 69-72.

Koyama, H., Yoshida, N., \& Amasaka, K. (2010). The A-mpm decision-making model for film project investment: a partnership with filmmakers. Proceedings of the 9th WSEAS International Conference on System Science and Simulation in Engineering, 215-220

Ohara, D., \& Yamada, M. (2009). A research on the movie poster created to enhance the viewing motivation: Using an eye mark recorder and statistical science. Aoyama Gakuin Universtiy's graduation thesis. (Unpublished)

Shinozaki, K., \& Shiizuka, H. (2007). Kansei forecasts of movie posters. Kogakuin University research report, 103, 203-207 (in Japanese)

Shu, G. M., \& Wei, Q. W. (2011). Effectively discriminating fighting shots in action movies. Journal of Computer Science and Technology, 26(1), 187-194. http://dx.doi.org/10.1007/s11390-011-9425-6

Takahashi, Y., Hasegawa, K., Sugiyama, K., \& Watanabe, M. (2000). A new movie summarization algorithm for effective movie selection: Toward human content interface design (4). Bulletin of Japanese Society for Science of Design, 142(1), 41-50 (in Japanese)

Tanimura, M. (2004). A Study of the behavior model with AIDA theory :Behavioral factor analysis as an example the TVCM and newspaper advertising of movies. Aoyama Gakuin Universtiy's graduation thesis. (Unpublished)

\section{Copyright Disclaimer}

Copyright reserved by the author(s).

This article is an open-access article distributed under the terms and conditions of the Creative Commons Attribution license (http://creativecommons.org/licenses/by/3.0/). 